\title{
Symptomatic endometriosis developing several years after menopause in the absence of increased circulating estrogen concentrations: a systematic review and seven case reports
}

\author{
Fernanda de Almeida Asencio', Helizabet Abdalla Ribeiro', Paulo Ayrosa Ribeiro', Mario Malzoni², Leila Adamyan³, \\ Anastasia Ussia, ${ }^{4,5}$, Victor Gomel ${ }^{6}$, Dan C. Martin ${ }^{7,8}$ and Philippe R. Koninckx $x^{4,5,9,10,11^{*}}$ (D)
}

\begin{abstract}
Background: To review women with symptomatic and clinically progressive endometriosis after menopause in the absence of estrogen intake or excessive systemic endogenous production.

Design: Seven case reports and a systematic review of the literature from 1995 till February 2018.

Results: Only 7 case reports from the authors and 29 cases from the literature described women with either cystic ovarian or deep endometriosis. Severity, symptoms, and localization are highly variable. No case report describes symptomatic superficial typical lesions. In 22 of 36 women (61\%), symptoms started more than 10 years after menopause.

Conclusions: Symptomatic and clinically progressive endometriosis after menopause in the absence of increased systemic estrogen concentrations or exogenous estrogen intake starts more than 10 years after menopause in the majority of women. This observation suggests that a genetic and/or epigenetic incident caused estrogen-independent progression, increased sensitivity to estrogens or increased local production of estrogens. This observation is important for understanding the pathophysiology of endometriosis, for the management of postmenopausal endometriosis, and for individualization of medical therapy of endometriosis since estrogen-independent endometriosis growth probably also occurs before menopause.
\end{abstract}

Keywords: Endometriosis, Pathophysiology of endometriosis, Postmenopausal endometriosis, Genetics, Epigenetics

\section{Introduction}

Symptomatic endometriosis starting several years after menopause in women not taking hormone replacement therapy (HRT) is a rare condition. Case reports only describe women with increasing pain, ovarian masses, or intestinal symptoms suspicious of a cancer. This is consistent with Kempers' [1] observations in 1960 on postmenopausal endometriosis. This was before estrogen assays, laparoscopy or ultrasound. Of all postmenopausal women operated for clinical symptoms between 1945

\footnotetext{
* Correspondence: pkoninckx@gmail.com

${ }^{4}$ Gruppo Italo Belga, Villa del Rosario, Rome, Italy

${ }^{5}$ Università Cattolica, Rome, Italy

Full list of author information is available at the end of the article
}

and 1958 in the Mayo clinic, endometriosis was found in 136 women, of whom 41 had symptoms compatible with endometriosis, 35 had an endometrioma, and 4 had progressive severe pain and/or bowel symptoms. The article in addition reported that in a control group of 50 hysterectomies, 8 atrophic endometriosis lesions were found.

Endometriosis is considered a hormonally responsive endometrium-like tissue where estrogens stimulate growth and progestins stop growth and eventually cause decidualization. It seems logical that after menopause endometriosis will become less active [2-4] since endometriosis is defined as (normal) endometrium-like tissue outside the uterus resulting from implantation of cells or endometrial fragments following retrograde menstruation [5]. However, the 
pathophysiology of endometriosis [6] is unclear and endometriosis may result from dislocation of basal endometrium [7] and metaplasia of peritoneal mesothelial cells [8] or of peritoneal or endometrial stem cells [9]. That endometriosis is phenotypically different from endometrium is according to the implantation/metaplasia theory, considered to be the consequence of the environmental factors in the peritoneal cavity, and of immunologic or of other predisposing factors.

The concept that endometriosis is a normal endometrium outside the uterus is not consistent with all clinical observations. The clinical, histologic, and immuno-cytochemical differences suggested that peritoneal, ovarian, and deep endometriosis are three different diseases [10]. The normal endometrium theory is not compatible with the clonal aspect of deep and cystic endometriosis [6]. Therefore, the endometriotic disease theory (EDT) $[6,11]$ and recently the genetic-epigenetic theory [12] suggest that the endometriotic cells are cells with a series of genetic or epigenetic transformations with epigenetic defined as "heritable changes in gene function that do not involve changes in the DNA sequence." These changes can occur in either mature adult or neonatal endometrium [13] following retrograde menstruation, or in stem/progenitor cells from the endometrium [13] or from the peritoneal cavity or even in bone marrow cells [14-16]. The specific combination of genetic and epigenetic incidents will determine the development into typical, cystic, or deep endometriosis. Subtle lesions are considered a mixture of implanted normal endometrium and of early endometriosis which after additional genetic and/or epigenetic incidents can develop into more severe lesions.

Stimulated by the observation of two women with progressively increasing symptoms of endometriosis after menopause in the absence of increased estrogen secretion or intake, we decided to perform a systematic review of symptomatic postmenopausal endometriosis in the absence of increased systematic endogenous estrogen production or estrogen intake. The aim was to establish its prevalence and clinical presentation.

\section{Materials and methods}

\section{Case reports and systematic review}

The aim of the study was to find women with progressively increasing symptoms caused by endometriosis after menopause and in the absence of estrogen intake or increased estrogen production. PubMed (comprising MEDLINE and Cochrane) and Scopus were searched according to the PRISMA checklist for publications in English between 1995 and February 2018 with "endometriosis" AND ("postmenopause" OR "menopause" OR "menopausal" OR "postmenopausal") in the title and for "endometriosis" AND "postmenopause" AND NOT "cancer" in the title, keywords, or abstract respectively.
The 102 and 241 articles found, respectively, were manually screened for the absence of increased systemic endogenous estrogen production or estrogen intake in women with progressively increasing symptoms after menopause likely caused by endometriosis. The 29 reports found were searched for the age when the diagnosis was made and for the number of years since surgical or spontaneous menopause. When the exact age of menopause was not available, we used 50 years (10 case reports, Table 1 , indicated as $* 50 *$ ) to calculate years since menopause. A variation of a few years or omitting these reports did not change the conclusions. Years since menopause was considered important since intermittently increased ovarian estrogen secretion can occur during the first years after menopause. In addition, the following items were recorded: previous surgery especially endometriosis surgery, drug intake, the presenting symptoms, the indication to start the investigation, pre-operative biochemistry and imaging, findings during surgery, and the final diagnosis by pathology. The systematic review was submitted to PROSPERO but not accepted since only a limited number of case reports were found. Our case reports were scrutinized in addition for the clinical exam with special attention to the presence or absence of vaginal atrophy, defined as an atrophic vaginal appearance with para-basal cells on a wet smear.

The quality of evidence described in these case reports [17] is high since the descriptions have comprehensive details written by clinicians noting the uncommon finding during surgery in postmenopausal women of severe endometriosis in the absence of increased circulating estrogen concentrations. However, the reports obviously carry a publication bias, since only women with symptoms sufficiently severe to perform surgery and in whom severe endometriosis was found were published. This review does not draw conclusions on the prevalence of symptomatic endometriosis after menopause, which is likely much higher. We know that unexpected endometriosis is frequent in women undergoing surgery after menopause [1].

We asked our colleagues (see the "Acknowledgments" section) with a known interest and a large experience of severe endometriosis surgery to retrieve all women in whom they had performed surgery for postmenopausal endometriosis without increased estrogen production or estrogen intake. This resulted in five additional cases and in a few cases of whom the records could not be retrieved.

\section{Types of endometriosis: definitions used}

Endometriosis, defined as "endometrial-like glands and stroma outside the uterus," can present as small microscopical lesions and macroscopically as subtle, typical, cystic, and deep lesions. Although the case reports of postmenopausal lesions described in this manuscript are 


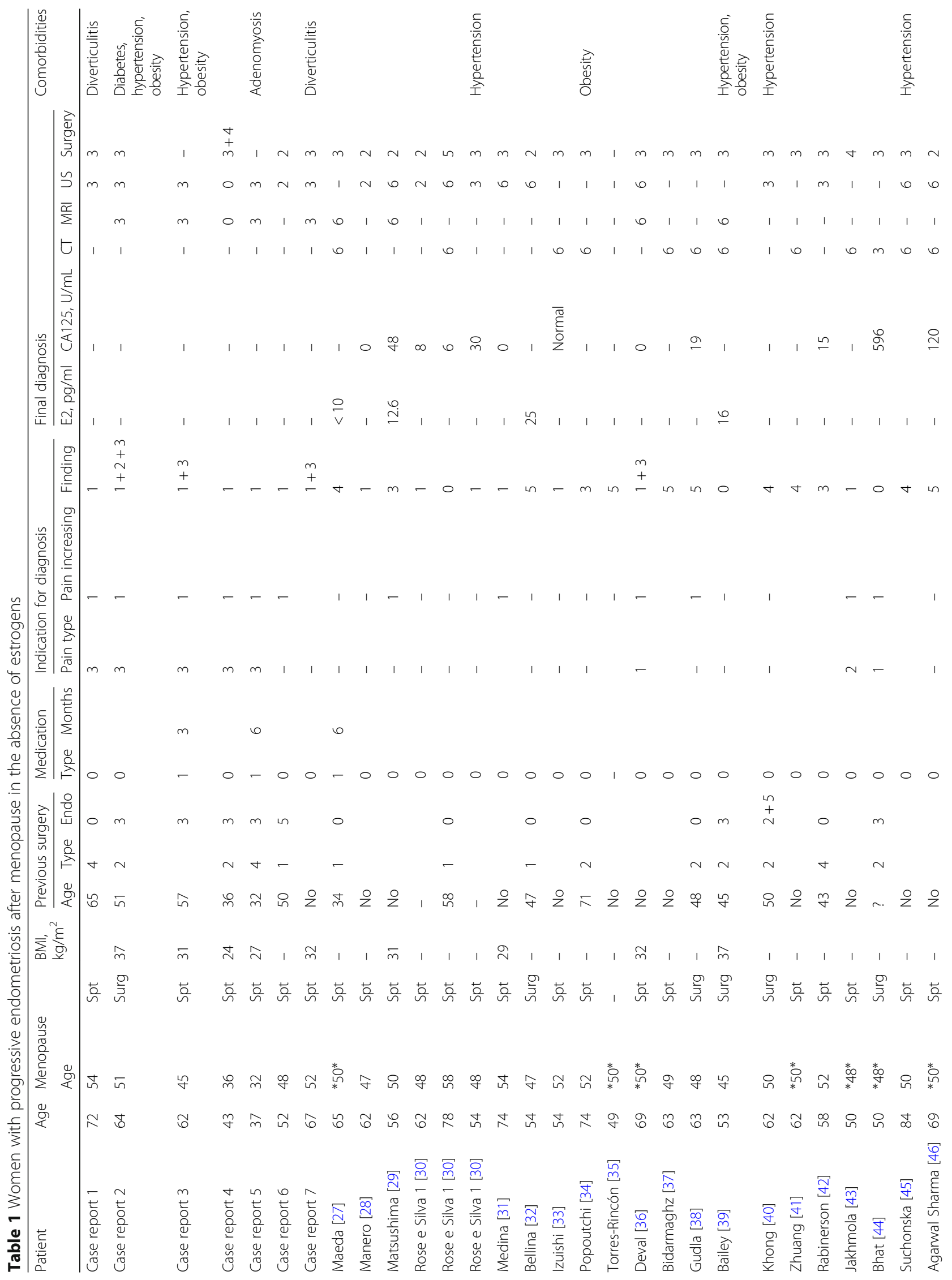




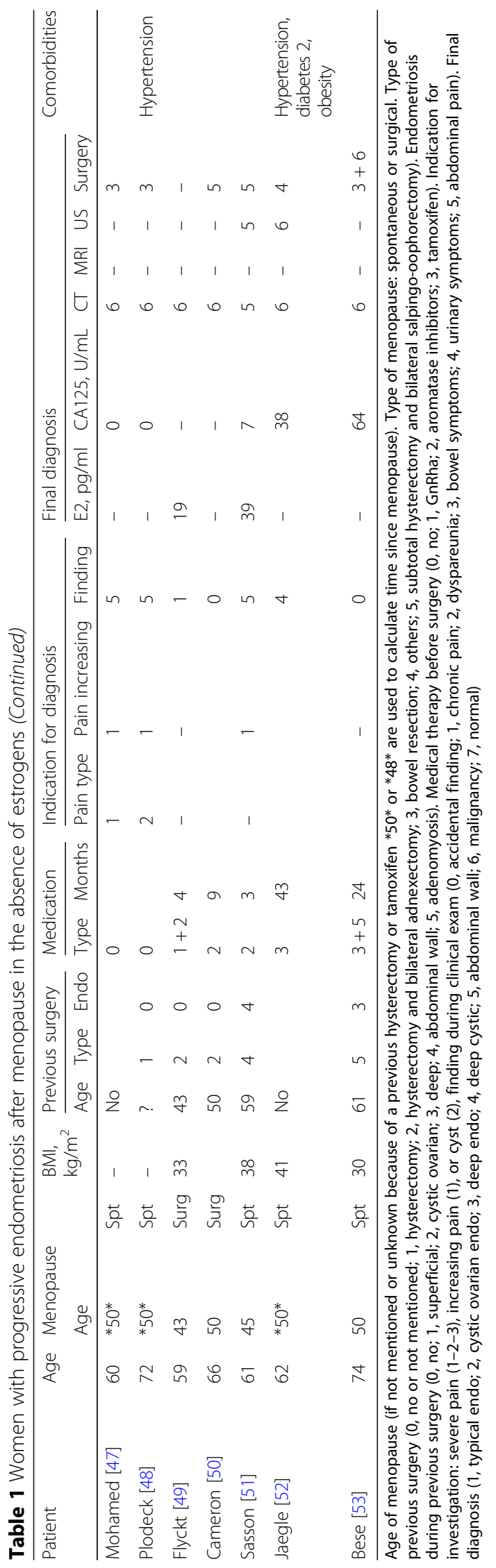


limited to cystic ovarian endometriosis and larger nodules of deep endometriosis, the definitions used in this manuscript are as follows. Typical lesions are superficial (less than $5 \mathrm{~mm}$ ) "powder burn" black dots in a fibrotic area. Subtle lesions are non-colored superficial lesions [18]. A discussion of the exact depth of subtle lesions, of their clinical significance [19], of the histological confirmation of biopsies [20], of Müllerianosis [21], and of the significance of larger areas with subtle lesions is beyond the scope of this article. Cystic endometrioses are the (larger) chocolate cysts of the ovary but not the smaller $(3-6 \mathrm{~mm})$ cysts under the peritoneum or close to the vagina in deep endometriosis. Deep endometriosis is defined as endometriosis deeper than $5 \mathrm{~mm}$ under the peritoneal surface [22]. A discussion of the limitations of depth to define deep endometriosis [12] is beyond the scope of this manuscript.

\section{Results}

Case reports of postmenopausal endometriosis in women without estrogen intake or signs of endogenous estrogen production

Only seven case reports could be collected, and ten surgeons did not remember having seen such cases.

\section{Case 1 (FA)}

A 72-year-old woman with spontaneous menopause at age 54 was referred for increasing pelvic pain. She had her menarche at 11 years and did not have antecedents of dysmenorrhea, dyspareunia, dysuria, dyschezia, endometriosis, nor infertility. Her medical, surgical, and gynecological history was uneventful except a diverticulitis and a volvulus 6 years before. She had never taken hormone replacement therapy (HRT), and the vaginal epithelium was atrophic. The gynecological exam and a transvaginal ultrasound confirmed a normal uterus and ovaries. However, it also showed a 1-cm arciform thickening at the insertion of the right uterosacral ligament and a $24-\mathrm{mm}$ nodule at $11 \mathrm{~cm}$ from the anus, on the recto-sigmoid, infiltrating the muscularis and affecting $15 \%$ of the circumference of the bowel. In the absence of other signs of malignancy, a laparoscopy was performed with a total hysterectomy, bilateral salpingo-oophorectomy, a segmental resection of the bowel, and a resection of the retro-cervical and right pararectal nodule. The pathology revealed active endometriotic glands and stroma in the retro-cervical and right pararectal nodules. The bowel lesion had fibrosis secondary to diverticulitis. Recovery was uneventful, and she was discharged on day 5 . After 4 weeks, she returned to her normal activities and is pain free.

\section{Case 2 (FA)}

A 64-year-old woman with spontaneous menopause at age 50 was referred for increasing pelvic pain, dyspareunia, dyschezia, and repetitive constipation which had started some 5 years ago. She was a G3 P1 A2 with menarche at 11 years of age and a BMI of 37. At age 51, she had been operated for abdominal pain, nausea, and a large endometriotic mass close to the left kidney with signs of renal failure. An abdominal total hysterectomy with bilateral salpingo-oophorectomy and left nephrectomy had confirmed the diagnosis of endometriosis. She never had taken HRT, and the vaginal epithelium was atrophic. Clinical exam revealed a large painful nodule. MRI showed two nodules: one large nodule in the pouch of Douglas, invading the vaginal cuff and left parametrium and extending up to the hypogastric plexus and the left sacral nerves ( 33 and S4); another nodule of $37 \times 32 \times 14 \mathrm{~mm}$ was seen in the rectum at $7.9 \mathrm{~cm}$ from the anal border, infiltrating the muscularis and submucosa. Transvaginal ultrasound examination confirmed a $35 \times 28 \times 23 \mathrm{~mm}$ nodule in the vaginal cuff and a $41 \times 10 \times 19 \mathrm{~mm}$ nodule in the recto-sigmoid, compromising $50 \%$ of the circumference, infiltrating the muscularis and submucosa at $9 \mathrm{~cm}$ from the anal border. A laparoscopic segmental bowel resection and resection of the deep endometriosis nodule invading the pelvic floor, the sacral nerves, and the left sciatic nerve confirmed the diagnosis of endometriosis. Postoperative recovery was uneventful, and she was discharged on day 5 and is pain free for 5 months.

\section{Case 3 (FA)}

A 62-year-old woman with spontaneous menopause at age 45 was seen for increasing severe pelvic pain $(10 / 10)$ and rectal pain with tenesmus. She was a P3G3 with menarche at age 13, a BMI of 31, and hypertension. She had not taken HRT since menopause, and the vagina was atrophic. Four years before, she had undergone a recto-sigmoidectomy with resection of an endometriosis nodule from the right uterosacral ligament and rectovaginal septum. Not responding to treatment with GnRha after surgery, she returned in 2017. At MRI, a retro-cervical nodule infiltrating the apical portion of the vagina and a nodule $(17 \times 06 \mathrm{~mm})$ in the sigmoid were found. Ultrasonography confirmed the rectovaginal $(15 \times 4 \mathrm{~mm})$ and the recto-sigmoid nodule $(17 \times 7 \times 3 \mathrm{~mm})$, compromising $25 \%$ of the circumference and infiltrating the muscularis. She is planned for surgery.

\section{Case 4 (AU)}

A 43-year-old woman with surgical menopause at age 36 visited for increasing severe pain symptoms for 2 years. She was a P2G2 with menarche at 11 years and a BMI of 24 . She had undergone a hysterectomy at age 34 for pain and a bilateral adnexectomy at age 36 for pain and endometriosis, after which she was pain free for 5 years. She had not taken HRT postoperatively, and her vagina was 
atrophic. The gynecological examination, MRI, and ultrasound were negative. A diagnostic laparoscopy revealed a large pelvic endometriosis plaque $(5 \times 4 \mathrm{~cm})$ with small cystic spots $(3-5 \mathrm{~mm})$ in the pouch of Douglas and a deep endometriosis $(1 \times 1 \mathrm{~cm})$ nodule around the right ureter, all of which were removed during the procedure. Pathology confirmed the diagnosis of endometriosis. The postoperative recovery was uneventful, and she is pain free for 1 year.

\section{Case 5 (AU)}

A 37-year-old woman with spontaneous menopause at age 32 visited for increasing pain symptoms for 1 year. She was a P0G0 with a menarche at age 9 and a BMI of 27 . At 23 years of age, she had undergone an adnexectomy for pain, and at age 27 , we removed a rectovaginal deep endometriosis nodule extending around the left ureter $(3 \times 2 \times 2 \mathrm{~cm})$ and a cystic ovarian endometriosis from the remaining ovary. At age 32 , a hysterectomy without ovariectomy was performed. Postoperatively, she became menopausal with increased FSH concentrations and a 17b-estradiol concentration of $80 \mathrm{pgr} / \mathrm{ml}$, which is insufficient for endometrial proliferation. Following hysterectomy, she was pain free for 4 years, after which she had progressively increasing pelvic pain on no hormonal therapy. Her clinical examination, MRI, and ultrasound demonstrated a $4 \times 4 \mathrm{~cm}$ deep endometriosis nodule of the cecum. In order to avoid a fourth surgical intervention, she was treated with GnRha without success. We plan to try an aromatase inhibitor.

\section{Case 6 (LA)}

A 52-year-old woman with spontaneous menopause at age 48 visited for increasing pain symptoms for 1 year. She was a P3G8. At age 50, she had undergone a total laparoscopic hysterectomy because of pain and an adenomyotic uterus. She had not taken HRT, and clinical examination demonstrated vaginal atrophy. A large cystic ovarian endometriosis was diagnosed. The pathology of the excised cyst confirmed the endometriosis. The postoperative recovery was uneventful, and she now is pain free for 4 months.

\section{Case 7 (MM)}

A 67-year-old woman with spontaneous menopause at age 52 was referred because of chronic pelvic pain, dyschezia, dyspareunia intermittent diarrhea since several years, and a suspicion of vaginal fornix endometriosis. She was a P0G0 with menarche at 12 years, a BMI of 32, and a history of sigmoid diverticulosis. She had not been taking HRT since menopause. The clinical examination revealed a vaginally visible retro-cervical nodule extending to the left parametrium. By ultrasonography, this nodule $(18 \times 11 \times 29 \mathrm{~mm})$ was confirmed together with another nodule $(16 \times 5 \times 12 \mathrm{~mm})$ at 8 -cm distance from the anal verge. In addition to adenomyosis, a thickened endometrial layer suggestive for a polypoid hyperplasia, normal ovaries, and severe adnexal adhesions were found. MRI confirmed the retro-para-cervical nodule. After confirmation of endometrial hyperplasia without atypia on endometrial biopsy, a laparoscopic adhesiolysis, total hysterectomy, bilateral salpingo-oophorectomy, ureterolysis with resection of the retro-para-cervical and vaginal bowel nodule, and shaving of a small rectal nodule were performed. Adenomyosis and deep infiltrating endometriosis were confirmed at histopathologic evaluation. The postoperative recovery was uneventful, and she was discharged on day 2. She is pain free for 2 years without signs of recurrence both clinically and at US.

\section{A systematic review of clinically progressive postmenopausal endometriosis without estrogen intake or increased production}

We did not review postmenopausal women who underwent surgery and in whom endometriosis was found in the absence of hormone replacement therapy. In this group of women, endometriosis was found in 2 to $5 \%$ [23]. The reported studies had either been published in 1955 and 1960 before the introduction of HRT [1], or the absence of HRT treatment had been explicitly stated [24]. The article of Kempers [1] described that of the 41 women after menopause, 25 had cystic ovarian endometriosis, 7 diffuse pelvic endometriosis, 8 intestinal, and 1 vaginal endometriosis. The prevalence seems to decrease with age since $5,11,13,7,2,2$, and 1 women were $45-49,50-54$, $55-59,60-64,65-69,70-74$, and $75-80$ years old respectively (Fig. 1). The number of years since menopause was not available. More recently, a review from Genua [25] confirmed that 69 out of 72 postmenopausal women who underwent surgery between 1998 and 2010 and in whom endometriosis was found had not taken hormone replacement therapy. The interval between menopause and surgery ranged from 1 to 32 years (median 6 years). Only $16.7 \%$ had a previous history of endometriosis, and the median BMI was $25.0 \mathrm{~kg} / \mathrm{m}^{2}$. The presenting symptoms were abnormal uterine bleeding in $26.4 \%$, abdominal pain in $26.4 \%$, rectal bleeding in $2.8 \%$, urogynecological dysfunction in $2.8 \%$, vaginal bleeding in $1.4 \%$, and an asymptomatic pelvic cyst in $40.3 \%$. The indications for surgery were ovarian cyst $(43.0 \%)$, ovarian cancer $(13.9 \%)$, endometrial cancer (13.9. \%), atypical endometrial hyperplasia (6.9\%), uterine myomas (6.9\%), tubo-ovarian abscess (4.2\%), sigmoid cancer (2.8\%), uterine prolapse (2.8\%), and cervical cancer, vaginal nodule, cholecystitis, and appendicitis (1.4\%). The type of endometriosis found was cystic ovarian endometriosis in $79.2 \%$, superficial endometriosis in $11.1 \%$, and endometriosis of the right parametrium, vagina, appendix, uterosacral ligament, and rectum in $5.6 \%$. 

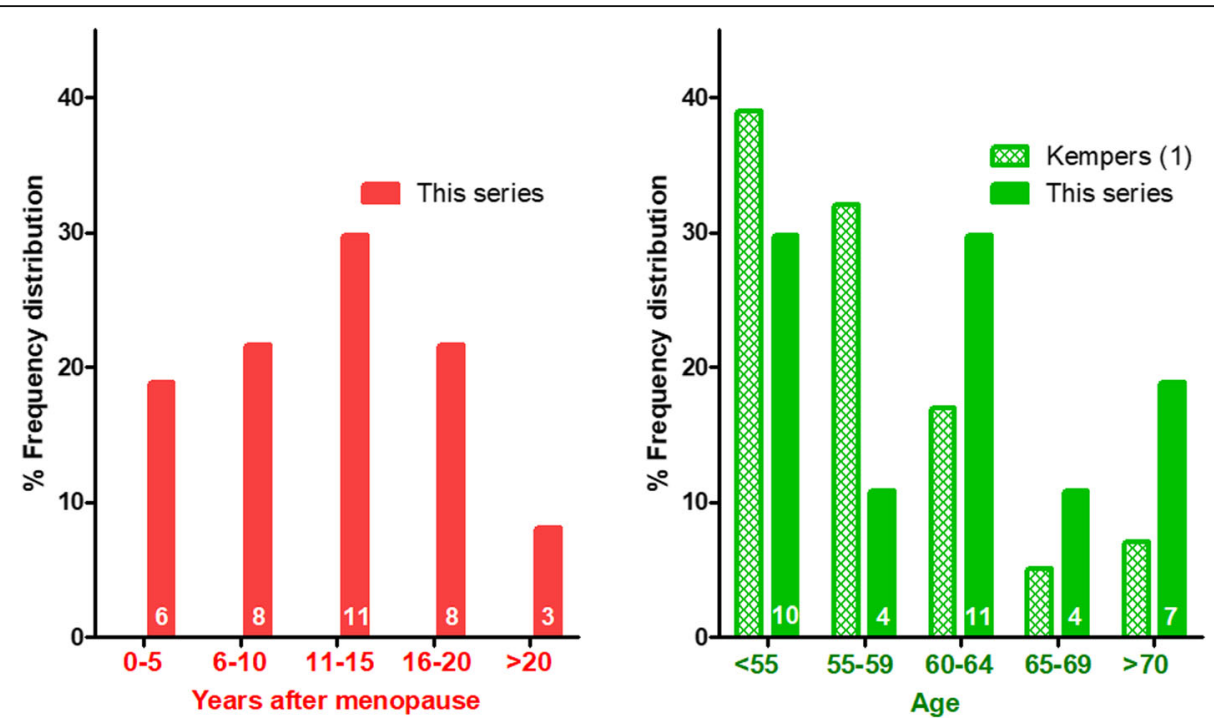

Fig. 1 Women with progressive and symptomatic endometriosis after menopause (this series) and women operated after menopause in whom endometriosis was found (Kempers). The frequency distribution of the years after menopause and/or the age of the women is indicated

Our review found 29 women (Table 1) with progressively increasing symptoms after menopause in whom endometriosis was diagnosed in the absence of HRT intake or an increased endogenous estrogen production. The symptoms are variable and comprise increasing pain with urinary symptoms [26], an asymptomatic cystic ovarian endometrioma or a cystic ovarian endometrioma with pain [27-31], a small bowel obstruction [32, 33], a rectovaginal deep endometriosis [29, 34], a sigmoid deep endometriosis [35], even a sigmoid obstruction more than 10 years after menopause [36], a deep endometriosis with progressive hydronefrosis [37], with renal failure [38] and with severe hypertension [39], urinary bleeding with an hydronephrosis and a polypoid intra-ureteral lesion [40], a vaginal endometriotic cyst [41], a lesion mimicking a bowel tumor [42], or an abdominal hemorrhage [43]. Some women were preoperatively suspected to have a cancer either an adenocarcinoma [44] or a disseminated ovarian cancer although during surgery only superficial endometriosis lesions together with bilaterally large endometriomas were found [45]. Even endometriosis suspicious of a gastric cancer [46] or of a pancreatic tumor in a 69-year-old woman [47], endometriosis with inferior vena cava involvement [48], and endometriosis in the abdominal wall were described. A nodular wall endometriosis developed in a woman without ovarian function treated with an aromatase inhibitor for breast cancer [49]. A new cystic endometrioma in the wall appeared spontaneously after the excision of two previous cysts. Plasma concentrations of $17 \mathrm{~b}$-estradiol were low $(20-40 \mathrm{pg} / \mathrm{ml})$; cyst fluid concentrations were $80 \mathrm{pg} / \mathrm{ml}$ returning to normal after GnRha treatment [50]. A series of reports describe progressive endometriosis in women taking tamoxifen [51-54].
Figure 1 illustrates that a majority of women were over 60 years old and more than 10 years after menopause.

The treatment of symptomatic postmenopausal endometriosis seems to be surgical excision. If this cannot be performed, a treatment with aromatase inhibitors was suggested [55].

\section{Discussion}

The article of Kempers [1] and the three reviews on endometriosis found in women undergoing surgery after menopause [23-25] describe postmenopausal women who did not take HRT. It is not surprising that the prevalence decreases with age (Fig. 1) since the age of menopause is variable and since ovarian activity can be intermittently increased in the first years after menopause. Unfortunately, the number of years since menopause is not always available. In addition, it is difficult to know whether the indication for surgery had been endometriosis, whether pain was caused by endometriosis, or whether endometriosis was a plausible explanation after surgery. They remain, however, remarkable documents with a detailed description of the clinical importance of endometriosis in women undergoing surgery after menopause [1]. The importance of postmenopausal endometriosis is also emphasized in this review of contemporary articles. Endometriosis was considered to be the cause of postmenopausal surgery in 13 women out of 516 surgeries by Scott and Te Linde [56] and in 37 out of 1000 by Henriksen [57]. To interpret these data collected more than 60 years ago, we should remember that this was before the introduction of hormone replacement therapy, estrogen assays, or ultrasound and with little knowledge of endocrinology such as 
peripheral conversion to estrogens and the intermittent and variable ovarian activity during the first years after menopause. This explains Kempers' conclusion that these women must have had estrogen production somewhere. He also clearly demonstrates that most women were young and (probably) early after menopause. Differences in life expectancy cannot explain the differences between the data in the Kempers' article and in this article since life expectancy after menopause was estimated to be 27 years in 1955 and 33 years today [58].

Our series describe women with progressive symptoms after menopause in whom endometriosis was diagnosed in the absence of estrogen intake or increased endogenous production. This is a rare condition, limited to case reports. Notwithstanding all limitations imposed by a recall bias, this also seems to be a rare condition when asking surgeons with a large experience in endometriosis surgery. The biphasic frequency distribution of age and years after menopause moreover suggests two different endometriosis populations. The high incidence of endometriosis in women younger than 55 years old or early after menopause and decreasing thereafter is compatible with an estrogenresponsive tissue. The second group after 60 years or more than 10 years after menopause can only be explained by circulating estrogen-independent progression of endometriosis or a change in estrogen sensitivity or local production of estrogens. In more than half of the women, symptoms started more than 10 years after menopause. That women clearly remember when symptoms started moreover suggests that the progression of these endometriosis lesions started abruptly at a specific point in time after menopause. This observation in postmenopausal women can be explained by the occurrence of a new genetic or epigenetic incident as postulated by the genetic-epigenetic theory to be the pathophysiology of endometriosis [12]. That such an incident is more likely to occur in women with a predisposition and with previous genetic-epigenetic incidents is supported by the observation that most of these women had been diagnosed with endometriosis before menopause. This genetic-epigenetic instability also is fully compatible with the rare occurrences of malignant transformation of endometriosis after menopause [59].

It is surprising that all reports of clinically progressive postmenopausal endometriosis lesions were either cystic ovarian endometriosis or deep endometriosis, without a single case report of clinically progressive superficial endometriosis. This contrasts with the high prevalence of typical lesions in premenopausal women who undergo a diagnostic laparoscopy for chronic pain. It is unclear, whether these women have less severe pain, and thus is not reported. The absence of reports thus can be a publication bias. An alternative explanation could be that after menopause typical lesions no longer cause pain. Only deep and cystic ovarian endometriosis seem to carry the risk that additional genetic or epigenetic incidents cause them to start proliferation in the absence of increased circulating estrogens.

Our series permits the conclusion that (some) cystic or deep endometriosis lesions can progress notwithstanding the low postmenopausal estrogen concentrations which are insufficient to stimulate the endometrium or to lubricate the vagina. Conversion of precursor steroids to estrogens in peripheral tissue, especially fat, might have played a role in some cases, as in the seventh case report with endometrial hyperplasia and obesity. In most cases, however, peripheral conversion as a cause of endometriosis progression is unlikely considering the absence of endometrial stimulation and the onset of symptoms many years after menopause in non-obese women. An alternative explanation could be a local estrogen production within the endometriosis lesions. Aromatase activity with local estrogen production and/or progesterone resistance in endometriosis lesions of young women are well known, although not yet described in postmenopausal endometriosis. The mechanism and pathways for this aromatase activity and local estrogen production and lack of apoptosis are moreover increasingly viewed as the result of genetic and/or epigenetic incidents or genetic polymorphism [2, 60-62]. The case report of the wall endometrioma in a postmenopausal woman [50] confirms aromatase activity because of the increased, although still below $100 \mathrm{pg} / \mathrm{ml}$, estrogen concentration in the cyst fluid.

These endometriosis lesions with progressive symptoms and starting often more than 10 years after menopause strongly suggest heterogeneity of cystic and deep endometriosis. Indeed, most cystic and deep endometriosis lesions are stimulated by estrogens and growth will be inhibited by progestins. This is moreover indirectly confirmed by the many reports of endometriosis in postmenopausal women taking hormone replacement therapy. However, some lesions grow in the absence of increased estrogen concentrations in plasma. The frequency of these circulating estrogen-independent lesions before menopause is unknown since they will only be diagnosed after menopause. This heterogeneity in cystic and deep endometriosis moreover is consistent with clinical observations during surgery. Heterogeneity of endometriosis lesions is also consistent with the variable response to medical therapy. Whereas the response of the endometrium to estrogens and progestins is predictable, pain relief of endometriosis by medical therapy is much more variable. The occasional progression of deep endometriosis [63] or the severe bleedings [64] during pregnancy also supports heterogeneity of lesions.

These rare symptomatic and progressive cases of endometriosis after menopause, starting more than 10 years after menopause, and the heterogeneity of cystic and deep endometriosis lesions and the heterogeneity in aromatase activity and progesterone resistance in endometriosis 
lesions can be explained with the genetic-epigenetic theory $[6,12]$, postulating a cumulative series of genetic or epigenetic incidents in the pathophysiology of endometriosis. Predisposition or susceptibility of endometriosis is considered to be caused by genetic and epigenetic incidents transmitted at birth. The transmitted incidents are insufficient to express the disease, but a woman with more severe transmitted incidents is at higher risk to develop the disease when additional incidents occur later. The many differences in the endometrium of women with and without endometriosis can be considered as the expression of these incidents transmitted at birth. The variable genetic and/or epigenetic incidents can explain that endometriosis is heterogeneous. It also can explain that the growth of endometriosis can occur notwithstanding low circulating estrogen concentrations as evidenced by this series and by endometriosis in a man [65] not taking estrogens (the other six reported cases of endometriosis were in men who had been taking estrogens for prostate cancer [66]). The onset of these progressive and symptomatic lesions more than 10 years after menopause suggests that new or additional incidents did occur. This poly-genetic and poly-epigenetic concept of pathophysiology of endometriotic disease also is fully compatible with recent histochemical profiling of endometriosis [67] and the induction of epigenetic changes in endometriosis [68].

Already in 1960 [1], Kempers described a series of women with active adenomyosis after menopause. Although the relationship between adenomyosis and endometriosis remains unclear, a similar pathogenesis of a combination of a series of genetic and/or epigenetic incidents can be postulated [69].

In conclusion, postmenopausal endometriosis progressing in the absence of estrogen intake or of a systemic increased production is an argument for the geneticepigenetic theory to explain the pathophysiology of endometriosis. These lesions thus should be considered a benign tumor and no longer as normal endometrium or as "normal" metaplastic cells with an abnormal behavior because of the environment or of the immunology. The direct clinical consequences of endometriosis progressing after menopause in the absence of increased estrogen concentrations are the heterogeneity of endometriosis lesions with a variable reaction during pregnancy and a variable response to medical therapy. Since this circulating estrogen-independent growth probably also occurs before menopause, women with medical therapy need an individual follow-up and therapy should be reconsidered when lesions grow. After menopause, it is important to recognize these lesions as different from a cancer.

\section{Conclusions}

Postmenopausal endometriosis can start to progress more than 10 years after menopause in the absence of estrogen intake or of a systemic increased production. This suggests that an acute genetic or epigenetic incident happened. The exact mechanism is not known, and it is unclear whether the growth of these lesions is estrogen independent or the consequence or an altered estrogen sensitivity or of an altered estrogen production within the lesion. It is unclear whether these are new lesions or a change in existing lesions. This observation can be explained by the genetic-epigenetic theory which considers the pathophysiology of endometriosis as a cumulative series of genetic or epigenetic incidents. That the specific set of incidents is specific for each lesion can explain the heterogeneity of endometriosis lesions. After menopause, only incidents which stimulate growth in the absence of increased circulating estrogens will become clinically symptomatic. These lesions thus should be considered a benign tumor and not as "normal" metaplastic cells with an abnormal behavior because of the environment or of the immunology.

\section{Acknowledgements \\ We thank Mauricio Abrao (Sao Paulo), Jacques Donnez (Belgium), Victor Gomel (Canada), Jörg Keckstein (Austria), Ceana and Camran Nezhat (USA), Antonio Setubal (Lisbon), Charles Koh (USA), Harry Reich and Assia Stepanian (USA) Arnaud Wattiez (Dubai) and Errico Zupi (Italy). Unfortunately they could not retrieve additional case reports.}

\section{Funding}

No funding

Availability of data and materials

Records of case reports are available with the authors as indicated

\section{Authors' contributions}

FAA, PK, and AU contributed to the conception and design of the study. FAA, PA, and PK contributed to the acquisition of the data. All authors contributed to the drafting and revision and final approval of the manuscript.

Ethics approval and consent to participate

Not applicable

Consent for publication

All authors agreed for publication

Competing interests

The authors declare that they have no competing interests.

\section{Publisher's Note}

Springer Nature remains neutral with regard to jurisdictional claims in published maps and institutional affiliations.

\footnotetext{
Author details

${ }^{1}$ Laparoscopy and Endometriosis Group Department, Santa Casa School of Medical Sciences of São Paulo, São Paulo, Brazil. 'Endoscopica Malzoni, Center for Advanced Endoscopic Gynecologic Surgery, Via C. Errico 2, 83100 Avellino, Italy. ${ }^{3}$ Department of Operative Gynecology, Federal State Budget Institution V. I. Kulakov Research Center for Obstetrics, Gynecology, and Perinatology, Ministry of Health of the Russian Federation, Moscow, Russia; and e Department of Reproductive Medicine and Surgery, Moscow State University of Medicine and Dentistry, Moscow, Russia. ${ }^{4}$ Gruppo Italo Belga, Villa del Rosario, Rome, Italy. ${ }^{5}$ Università Cattolica, Rome, Italy. ${ }^{6}$ Department of Obstetrics and Gynecology, University of British Columbia Women's Hospital, Vancouver, BC, Canada. ${ }^{7}$ School of Medicine, University of Tennessee Health Science Center, Memphis, TN, USA. ${ }^{8}$ Institutional Review Board, Virginia Commonwealth University, Richmond, VA, USA. ${ }^{9} \mathrm{KU}$ Leuven,
} 
Vuilenbos 2, 3360 Bierbeek, Belgium. ${ }^{10}$ University of Oxford, Oxford, UK.

${ }^{11}$ Moscow State University, Moscow, Russia.

\section{Received: 23 September 2018 Accepted: 10 January 2019} Published online: 15 February 2019

\section{References}

1. Kempers RD, Dockerty MB, Hunt AB, Symmonds RE (1960) Significant postmenopausal endometriosis. Surg Gynecol Obstet 111:348-356

2. Bulun SE (2009) Endometriosis. N Engl J Med 360:268-279

3. Cumiskey J, Whyte P, Kelehan P, Gibbons D (2008) A detailed morphologic and immunohistochemical comparison of pre- and postmenopausal endometriosis. J Clin Pathol 61:455-459

4. Streuli I, Gaitzsch H, Wenger JM, Petignat P (2017) Endometriosis after menopause: physiopathology and management of an uncommon condition. Climacteric 20:138-143

5. Sampson JA (1925) Heterotopic or misplaced endometrial tissue. Am J Obstet Gynecol 10:649-664

6. Gordts S, Koninckx P, Brosens I (2017) Pathogenesis of deep endometriosis. Fertil Steril 108:872-885

7. Leyendecker G, Herbertz M, Kunz G, Mall G (2002) Endometriosis results from the dislocation of basal endometrium. Hum Reprod 17:2725-2736

8. Gruenwald P (1942) Origin of endometriosis from the mesenchyme of the celomic walls. Am J Obstet Gynecol 44:470-474

9. Cousins FL, DF O, Gargett CE (2018) Endometrial stem/progenitor cells and their role in the pathogenesis of endometriosis. Best Pract Res Clin Obstet Gynaecol 50:12

10. Nisolle M, Donnez J (1997) Peritoneal endometriosis, ovarian endometriosis, and adenomyotic nodules of the rectovaginal septum are three different entities. Fertil Steril 68:585-596

11. Koninckx PR, Barlow D, Kennedy S (1999) Implantation versus infiltration: the Sampson versus the endometriotic disease theory. Gynecol Obstet Investig 47(Suppl 1):3-9

12. Koninckx PR, Ussia A, Adamyan L, Wattiez A, Gomel V, Martin D (2018) Pathogenesis of endometriosis: the genetic-epigenetic theory. Fertil Steril in press

13. Puttemans P, Benagiano G, Gargett C, Romero R, Guo SW, Brosens I (2017) Neonatal uterine bleeding as a biomarker for reproductive disorders during adolescence: a worldwide call for systematic registration by nurse midwife. J Matern Fetal Neonatal Med 30:1434-1436

14. Sandoval P, Jimenez-Heffernan JA, Guerra-Azcona G, Perez-Lozano ML, Rynne-Vidal A, Albar-Vizcaino P, Gil-Vera F, Martin P, Coronado MJ, Barcena C, Dotor J, Majano PL, Peralta AA, Lopez-Cabrera M (2016) Mesothelial-to-mesenchymal transition in the pathogenesis of post-surgical peritoneal adhesions. J Pathol 239:48-59

15. Cheng Y, Li L, Wang D, Guo Q, He Y, Liang T, Sun L, Wang X, Cheng Y, Zhang G (2017) Characteristics of human endometrium-derived mesenchymal stem cells and their tropism to endometriosis. Stem Cells Int 2017:4794827

16. Lucas PA (2007) Stem cells for mesothelial repair: an understudied modality. Int J Artif Organs 30:550-556

17. Guyatt GH, Oxman AD, Kunz R, Woodcock J, Brozek J, Helfand M, Alonso-Coello P, Glasziou P, Jaeschke R, Akl EA, Norris S, Vist G, Dahm P, Shukla VK, Higgins J, Falck-Ytter Y, Schunemann HJ (2011) GRADE guidelines: 7. Rating the quality of evidence--inconsistency. J Clin Epidemiol 64:1294-1302

18. Martin DC, Hubert GD, Levy BS (1989) Depth of infiltration of endometriosis. J Gynecol Surg 5:55-60

19. Koninckx PR, Donnez J, Brosens I (2016) Microscopic endometriosis: impact on our understanding of the disease and its surgery. Fertil Steril 105:305-306

20. Martin DC (2003) Endometriosis: correlation between histologic and visual findings at laparoscopy. Am J Obstet Gynecol 188:1663-1664

21. Batt RE, Smith RA, Buck Louis GM, Martin DC, Chapron C, Koninckx PR, Yeh J (2007) Mullerianosis. Histol Histopathol 22:1161-1166

22. Koninckx PR, Martin DC (1992) Deep endometriosis: a consequence of infiltration or retraction or possibly adenomyosis externa? Fertil Steril 58:924-928

23. Oxholm D, Knudsen UB, Kryger-Baggesen N, Ravn P (2007) Postmenopausal endometriosis. Acta Obstet Gynecol Scand:1-7. 87:1158-64

24. Punnonen R, Klemi PJ, Nikkanen V (1980) Postmenopausal endometriosis. Eur J Obstet Gynecol Reprod Biol 11:195-200
25. Morotti M, Remorgida V, Venturini PL, Ferrero S (2012) Endometriosis in menopause: a single institution experience. Arch Gynecol Obstet 286:1571-1575

26. Maeda T, Uchida Y, Nakajima F (2009) Vesical endometriosis following the menopause. Int Urogynecol J Pelvic Floor Dysfunct

27. Manero MG, Royo P, Olartecoechea B, Alcazar UL (2009) Endometriosis in a postmenopausal woman without previous hormonal therapy: a case report. J Med Case Rep 3:135

28. Matsushima T, Asakura H (2016) Huge ovarian endometrioma that grew after menopause: case report. J Obstet Gynaecol Res 42:350-352

29. Rosa e Silva JC, Carvalho BR, Barbosa HF, Poli-Neto OB, Rosa e Silva AC, Candido dos Reis FJ, Nogueira AA (2008) Endometriosis in postmenopausal women without previous hormonal therapy: report of three cases. Climacteric 11:525-528

30. Medina N, Martín A, Guillén V, Andújar M, García JA (2005) Ovarian endometrioma in a postmenopausal woman unrelated to neoplasia or exogenous hormone therapy. Progresos de Obstetricia y Ginecologia 48:150-153

31. Bellina JH, Schenck D (2000) Large postmenopausal ovarian endometrioma. Obstet Gynecol 96:846

32. Izuishi K, Sano T, Shiota A, Mori H, Ebara K (2015) Small bowel obstruction caused by endometriosis in a postmenopausal woman. Asian J Endosc Surg 8:205-208

33. Popoutchi P, Lemos CR, Silva JC, Nogueira AA, Feres O, Rocha JJ (2008) Postmenopausal intestinal obstructive endometriosis: case report and review of the literature. Sao Paulo Med J 126:190-193

34. Torres-Rincón RA, Moreno-Rojas A, Salinas-Parra C (2017) Endometriosis of the cecum in a postmenopausal women: case report and literature review. latreia 30:333-339

35. Deval B, Rafii A, Felce DM, Kermanash R, Levardon M (2002) Sigmoid endometriosis in a postmenopausal woman. Am J Obstet Gynecol 187:1723-1725

36. Bidarmaghz B, Shekhar A, Hendahewa R (2016) Sigmoid endometriosis in a post-menopausal woman leading to acute large bowel obstruction: a case report. Int J Surg Case Rep 28:65-67

37. Gudla VR, Tangudu S (2012) Postmenopausal endometriosis with ureteric involvement. Radiol Case Rep 7:607

38. Bailey AP, Schutt AK, Modesitt SC (2010) Florid endometriosis in a postmenopausal woman. Fertil Steril 94(2769):e2761-e2764

39. Khong SY, Lam A, Coombes G, Ford S (2010) Surgical management of recurrent ureteric endometriosis causing recurrent hypertension in a postmenopausal woman. J Minim Invasive Gynecol 17:100-103

40. Zhuang L, Eisinger D, Jaworski R (2017) A case of ureteric polypoid endometriosis presenting in a post-menopausal woman. Pathology

41. Rabinerson D, Avrech O, Kaplan B, Braslavsky D, Goldman GA, Neri A (1996) Endometrioma of the vagina in menopause. Acta Obstet Gynecol Scand 75:506-507

42. Jakhmola CK, Kumar A, Sunita BS (2016) Expect the unexpected: endometriosis mimicking a rectal carcinoma in a post-menopausal lady. J Minim Access Surg 12:179-181

43. Bhat RA, Teo M, Bhat AK (2014) Endometriosis after surgical menopause mimicking pelvic malignancy: surgeons' predicament. Oman Med J 29:226-231

44. Suchonska B, Gajewska M, Zygula A, Wielgos M (2018) Endometriosis resembling endometrial cancer in a postmenopausal patient. Climacteric 21:88-91

45. Agarwal Sharma R, Lee EY, Vardhanabhuti V, Khong PL, Ngu SF (2016) Unusual case of postmenopausal diffuse endometriosis mimicking metastastic ovarian malignancy. Clin Nucl Med 41:e120-e122

46. Mohamed AAA, Selim YARM, Arif MA, Albroumi SA (2016) Gastric wall endometriosis in a postmenopausal woman. Egypt J Radiol Nucl Med 47:1783-1786

47. Plodeck V, Sommer U, Baretton GB, Aust DE, Laniado M, Hoffmann RT, Platzek I (2016) A rare case of pancreatic endometriosis in a postmenopausal woman and review of the literature. Acta Radiol Open 5:2058460116669385

48. Flyckt R, Lyden S, Roma A, Falcone T (2011) Post-menopausal endometriosis with inferior vena cava invasion requiring surgical management. Hum Reprod 26:2709-2712

49. Cameron M, Westwell S, Subramanian A, Ramesar K, Howlett D (2017) Postmenopausal cutaneous endometriosis: mimicking breast metastasis. Breast J 23:356-358 
50. Sasson IE, Taylor HS (2009) Aromatase inhibitor for treatment of a recurrent abdominal wall endometrioma in a postmenopausal woman. Fertil Steril 92:1170-1174

51. Jaegle WT, Barnett JC, Stralka BR, Chappell NP (2017) Polypoid endometriosis mimicking invasive cancer in an obese, postmenopausal tamoxifen user. Gynecol Oncol Rep 22:105-107

52. Bese T, Simsek Y, Bese N, Ilvan S, Arvas M (2003) Extensive pelvic endometriosis with malignant change in tamoxifen-treated postmenopausal women. Int J Gynecol Cancer 13:376-380

53. Ismail SM, Maulik TG (1997) Tamoxifen-associated post-menopausal endometriosis. Histopathology 30:187-191

54. Buckley CH (1997) Tamoxifen-associated postmenopausal endometriosis. Histopathology 31:296

55. Polyzos NP, Fatemi HM, Zavos A, Grimbizis G, Kyrou D, Velasco JG, Devroey P, Tarlatzis B, Papanikolaou EG (2011) Aromatase inhibitors in post-menopausal endometriosis. Reprod Biol Endocrinol 9:90

56. Scott RB, Te Linde RW (1950) External endometriosis-the scourge of the private patient. Ann Surg 131:697-720

57. Henriksen E (1955) Endometriosis. Am J Surg 90:331-337

58. Glei DA, Mesle F, Vallin J (2010) Diverging trends in life expectancy at age 50: a look at causes of death. In: Crimmins EM, Preston SH, Cohen B (eds) International differences in mortality at older ages: dimensions and sources, National Academies Press (US), Washington (DC)

59. Taylor AA, Kenny N, Edmonds S, Hole L, Norbrook M, English J (2005) Postmenopausal endometriosis and malignant transformation of endometriosis: a case series. Gynecol Surg 2:135-137

60. Bulun SE, Monsivais D, Kakinuma T, Furukawa Y, Bernardi L, Pavone ME, Dyson M (2015) Molecular biology of endometriosis: from aromatase to genomic abnormalities. Semin Reprod Med 33:220-224

61. Dyson MT, Roqueiro D, Monsivais D, Ercan CM, Pavone ME, Brooks DC Kakinuma T, Ono M, Jafari N, Dai Y, Bulun SE (2014) Genome-wide DNA methylation analysis predicts an epigenetic switch for GATA factor expression in endometriosis. PLoS Genet 10:e1004158

62. Xue Q, Zhou YF, Zhu SN, Bulun SE (2011) Hypermethylation of the CpG island spanning from exon II to intron III is associated with steroidogenic factor 1 expression in stromal cells of endometriosis. Reprod Sci 18:1080-1084

63. Setubal A, Sidiropoulou Z, Torgal M, Casal E, Lourenco C, Koninckx P (2014) Bowel complications of deep endometriosis during pregnancy or in vitro fertilization. Fertil Steril 101:442-446

64. Brosens IA, Lier MC, Mijatovic V, Habiba M, Benagiano G (2016) Severe spontaneous hemoperitoneum in pregnancy may be linked to in vitro fertilization in patients with endometriosis: a systematic review. Fertil Steril 106:692-703

65. Giannarini G, Scott CA, Moro U, Grossetti B, Pomara G, Selli C (2006) Cystic endometriosis of the epididymis. Urology 68:203

66. Fukunaga M (2012) Paratesticular endometriosis in a man with a prolonged hormonal therapy for prostatic carcinoma. Pathol Res Pract 208:59-61

67. Liu X, Zhang Q, Guo SW (2018) Histological and immunohistochemical characterization of the similarity and difference between ovarian endometriomas and deep infiltrating endometriosis. Reprod Sci 25:329-340

68. Zhang Q, Dong P, Liu X, Sakuragi N, Guo SW (2017) Enhancer of Zeste homolog 2 (EZH2) induces epithelial-mesenchymal transition in endometriosis. Sci Rep 7:6804

69. Koninckx PR, Ussia A, Zupi E, Gomel V (2018) The relationship of endometriosis and adenomyosis. JMIG in press

\section{Submit your manuscript to a SpringerOpen ${ }^{\circ}$ journal and benefit from:}

- Convenient online submission

- Rigorous peer review

- Open access: articles freely available online

- High visibility within the field

- Retaining the copyright to your article

Submit your next manuscript at $\boldsymbol{\nabla}$ springeropen.com 